\title{
O PERFIL DA INOVAÇÃO NA INDÚSTRIA BRASILEIRA
}

\section{THE INNOVATION PROFILE OF BRAZILIAN INDUSTRY}

\author{
David Ferreira Lopes Santos ${ }^{1}$ \\ ${ }^{1}$ Universidade Estadual Paulista - UNESP - Jaboticabal/SP - Brasil \\ david.lopes@fcav.unesp.br
}

\begin{abstract}
Resumo
Este estudo examina o comportamento dos esforços em inovação na indústria brasileira a partir dos resultados da PINTEC/IBGE nos anos 2000, 2003 e 2005. Tomou-se como referencial o modelo do Índice Brasil de Inovação (FURTADO e QUADROS, 2006), em especial, o indicador agregado de esforço (IAE). A partir de uma análise descritiva das informações agregadas da PINTEC e de uma amostra média selecionada de 1.620 empresas, foi possível constatar que o perfil de inovação da indústria brasileira é voltado para o acompanhamento/modernização tecnológica, tendo em vista, que o principal investimento em inovação ocorre em Aquisição de Máquinas, de modo que recursos destinados à $P \& D$ Interno são modestos e aqueles orientados ao desenvolvimento de Conhecimento e Pesquisa externa à empresa são ínfimos. Não obstante, a participação de mestres e doutores em pesquisa na indústria nacional é inferior a $0,5 \%$ do efetivo total. Desta forma, constata-se que o perfil da indústria nacional não é voltado à inovação, mas ao acompanhamento desta.
\end{abstract}

Palavras-chave: inovação; indústria brasileira; PINTEC/IBGE.

\section{Introdução}

Pesquisas têm sido empreendidas no Brasil com o objetivo de mapear o esforço inovador do país, seja no âmbito da firma, ou do agregado nacional, destacam-se nessa seara os trabalhos pioneiros de Marcovitch (1978), Paulinyi (1990), Matesco (1993), Arruda (1994) e Andreassi (1999), todos anteriores a publicação sistematizada do Instituto Brasileiro de Geografia e Estatística (IBGE) sobre Pesquisa de Inovação Tecnológica (PINTEC) das empresas instaladas em território nacional.

A partir da base de dados resultante da PINTEC, sendo a primeira edição em 2000 (PINTEC, 2002), diferentes trabalhos foram produzidos no intuito de avaliar a capacidade de inovar e os reflexos destes investimentos nos resultados das empresas e do próprio país, relacionam-se, por exemplo, Morganti (2005), Sbragia (2006), Arruda, Vermulm e Hollanda (2006), Marques (2007) e Santos (2009) como exemplos de investigações quanto à condição de inovação do país. 
Em paralelo, merece destaque à criação em 2006 do Índice Brasil de Inovação (IBI) pelo Departamento de Política Científica da Universidade Estadual de Campinas. O índice é formado pela composição de dois indicadores IAE e IAR que representam o esforço para inovar e os resultados alcançados com a inovação, respectivamente (FURTADO e QUADROS, 2006).

Assim, tomou-se como base a estrutura do Indicador Agregado de Esforço (IAE) como modelo para estabelecer o perfil da inovação, no que tange a capacidade de inovar da indústria brasileira.

Tem-se como pacífico a importância da inovação para o país, ou melhor, de um Sistema Nacional de Inovação (SNI), como defende Sbragia (2006); essa importância deve-se aos reflexos da inovação na competitividade das empresas e no desenvolvimento da sociedade em todas as dimensões (DOSI, 2000). As questões que hoje ensejam esse tema dizem respeito à estrutura, dinâmica, mecanismos de quantificação e valoração, modelos de comparação, avaliação de eficácia, entre outros (TIDD, BESSANT, PAVITT, 2008).

Nesse contexto, insere-se essa pesquisa, cujo foco é analisar descritivamente os resultados agregados das edições 2000, 2003 e 2005 da PINTEC/IBGE, a fim, de verificar o perfil da inovação da indústria nacional.

Ressalta-se que após a edição de 2005, houve a edição 2008, publicada em 2010, cujos dados não estavam disponíveis no levantamento da base de informações junto ao IBGE para esta pesquisa. Contudo, tem-se um horizonte temporal importante na economia brasileira, pois marca a consolidação da estabilização da moeda e, o maior vigor econômico do país, com efeito, poder-se-á observar, longitudinalmente, o perfil do esforço em inovação da indústria brasileira nos primeiros cinco anos deste século.

Não obstante, a motivação para realização dessa pesquisa foi identificar, e, em certa medida, compreender onde o industriário nacional concentrou seus esforços em inovação e qual a estrutura desta frente à dimensão dessas empresas?

Por derradeiro, alçaram-se como objetivos:

a) Discutir a capacidade de inovar das empresas, segundo a teoria da inovação, a partir, do modelo proposto pelo IBI;

b) Consolidar os microdados das pesquisas publicadas pela PINTEC/IBGE de forma a facilitar a solução do problema proposto.

No intuito de melhor organizar a pesquisa realizada, estruturou-se este artigo da seguinte forma: a próxima seção traz os fundamentos teóricos que sustentam a fundamentação do tema e a discussão proposta no primeiro objetivo. Na sequência, abordam-se os procedimentos 
metodológicos adotados para a realização da pesquisa empírica, bem como, as limitações da análise. A quarta seção apresenta os resultados da investigação, para então nas considerações finais, propor uma perspectiva da capacidade de inovar da indústria nacional para o período, bem como proposições para pesquisas futuras. As referências bibliográficas encerram o artigo.

\section{Fundamentos teóricos}

A literatura que abarca a Teoria da Inovação é uníssona em creditar aos trabalhos de Schumpeter e posteriormente aos autores denominados neo-schumpteriados os precursores deste campo do conhecimento (DOSI, 2000) e (SANTOS, 2009). Todavia, a despeito, do tronco comum sedimentado nos trabalhos de Schumpeter, inúmeras pesquisas têm reclamado diferentes conceitos e características à inovação, portanto, faz-se necessário um delineamento da conceituação a ser assumido neste artigo.

Sem demérito a outras definições, adotou-se neste trabalho o entendimento de Grupp (1998) para inovação. O autor ressalta as diferenças nas terminologias utilizadas que em muitas oportunidades assumem inadvertidamente o que se propõe como inovação. Para Grupp (1998), a inovação quando considerada como um verbo - inovar - deve ser entendida por um processo orientado para resultados que podem ser expressos por novos produtos, novos sistemas de produção, transporte, sistema de gestão, desenvolvimento de novas fontes de suprimento de matéria prima e novos mercados, de forma que estas inovações proporcionem resultados financeiros, conhecidos em Schumpeter (1985) como rents de inovação.

Grupp (1998) esclarece que a inovação é uma conseqüência do desenvolvimento da ciência e da tecnologia e cujos resultados são alcançados, principalmente, por meio de uma expressiva atividade de Pesquisa e Desenvolvimento (P\&D). Neste sentido, a inovação no âmbito da empresa é materializada quando há o efetivo surgimento de algo que proporcione rents, todavia, o seu processo depende substancialmente de investimentos realizados em $\mathrm{P} \& \mathrm{D}$ e em áreas que sejam capazes de entregar aplicações ao domínio científico e tecnológico existente.

As inovações podem, ainda, serem classificadas como incrementais ou radicais, sendo a primeira alterações marginais nos produtos ou processos, e a segunda, alteraçoes substanciais que envolvem mudança no paradigma tecnológico vigente (SCHUMPETER, 1985). Para Schumpeter, são as inovações radicais as "molas propulsoras" do sistema capitalista.

As inovações (radicais ou incrementais) ainda podem ocorrer no produto ou processo de produção, não existindo na teoria, um consenso sobre qual orientação é a mais efetiva (SANTOS, 2009).

A perspectiva do resultado da inovação enquanto "aplicação econômica", também é defendia por Sundbo (1998), Soete e Freeman (1999) e Dosi (2000). Essa orientação, é de suma 
importância no Brasil, onde segundo constatação de Sbragia (2006) 72\% dos cientistas e engenheiros dedicados à pesquisa estão nas universidades, cuja aproximação com as cadeias produtivas, ainda é distante.

O crescente interesse das empresas em inovar está associado a sua necessidade de buscar ou defender um posicionamento competitivo no mercado globalizado (DOSI e NELSON, 1994). A estratégia das firmas, poderá ser reativa, isto é, copiando inovações propostas por concorrentes ou pró-ativas quando elas são responsáveis pela introdução de inovações no mercado (MANUAL DE OSLO, 2004). Todavia, a decisão de incorporar a estratégia de inovação no planejamento das organizações não é trivial, o processo de inovar requer esforço financeiro, humano e gerencial e os resultados são incertos e quando ocorrem, aparecem no longo prazo (DOSI, 2000), (TIDD, BESSANT e PAVITT, 2008). Ademais, a estrutura de inovação não é restrita as fronteiras da firma, ela se expande e coaduna com o ambiente que a cerca (MANGEMATIN e MANDRAN, 2002), (LEIPONEN, 2002) e (HINLOOPEN, 2003).

Por isso, as empresas precisam ter capacidade para absorver o conhecimento que está sendo produzido nas mais diversas instituições, pois a integração com diferentes fontes de conhecimento, a criação de um ambiente interno propício à inovação e o fortalecimento da cultura empreendedora reforçam os pressupostos schumpeterianos e corroboram as considerações encontradas em pesquisas acadêmicas (HINLOOPEN, 2003).

Assume-se, portanto, que a inovação pode ser compreendida como um processo, cujo objetivo para as empresas é a criação de valor ao negócio. Essa possibilidade só é possível quando os mercados são extremamente dinâmicos, ocasionados por ciclos de vida de produtos cada vez mais curtos oriundos das inovações radicais (SCHUMPETER, 1985). Esses postulados lançam as empresas em estruturas de mercado voláteis, caracterizadas pela constante alteração do comportamento dos agentes, exigindo dos pesquisadores e gestores das firmas novas premissas econômicas, pois o cerne dos fundamentos das empresas não é mais a busca pelo equilíbrio, mas sim, o interesse no rompimento das inércias tecnológicas existentes e por tanto nas estruturas de mercado.

Observa-se, portanto, a influência da corrente Evolucionária da economia, onde o foco da análise é a importância da mudança tecnológica e a diversidade do comportamento das firmas, o que permite um novo entendimento do desempenho das organizações (SILVA, 2004). Nessa esteira, Hu (2003) assevera sobre a melhor compreensão das organizações, quando estas são consideradas como entidades heterogeneas na sua forma de gestão e estrutura de recursos, portanto, devem apresentar diferenças na capacidade de inovação.

No pensamento evolucionário os conceitos de equilíbrio e otimização são refutados, pois o processo de evolução é por natureza path depence, sendo assim, diversos equilíbrios e estágios de 
equilíbrio são permitidos. A possibilidade em visualizar o avanço tecnológico como motor do desenvolvimento econômico acontecendo dentro das instituições é uma das premissas centrais da corrente evolucionária, entretanto, exportar essa perspectiva para o ambiente macro num modelo formalizado concentra o maior desafio da teoria evolucionária (CONCEIÇÃO, 2002).

Destarte, as organizações são compreendidas dentro de um sistema, onde a capacidade de tomar decisões relativas às suas estratégias, adaptação às mudanças ambientais e desenvolvimento tecnológico implicarão no seu nível de rentabilidade e por conseqüência na sua permanência no mercado, "a vida do homem em sociedade, assim como a vida de outras espécies, é uma luta pela existência e, conseqüentemente, é um processo de seleção adaptativa. A evolução da estrutura social tem sido um processo de seleção natural de instituições." (Veblen, 1899, p. 188, apud, Hodgson, 1993, p. 17).

Nestes princípios estão sustentadas as bases da teoria da inovação que tem como principais expoentes na atualidade Christopher Freeman, Giovanni Dosi, Keith Pavitt, David J. Teece, Richard Nelson e Sidney Winter. Nos trabalhos destes autores verifica-se um direcionamento comum das premissas utilizadas que tem servido para balizar as pesquisas atuais.

\title{
2.1. Índice Brasil de inovação
}

No interesse de mensurar a inovação nas empresas nacionais, surge o IBI desenvolvido pelo Departamento de Política Científica e Tecnológica do Instituto de Geociências da Universidade Estadual de Campinas (FURTADO, QUADROS, 2006).

\begin{abstract}
A proposta do IBI é criar um índice de inovação das empresas do setor industrial para ordená-las de acordo com seu grau de inovação (...) a idéia de criar um indicador agregado de inovação parte da premissa de que o processo de inovação é complexo e multifacetado, devendo, portanto seu índice compreender vários indicadores parciais referentes a dimensões específicas-chaves desse processo. Duas dimensões destacam-se nessa variedade: de um lado a necessidade de incluir os esforços realizados pelas empresas para inovar, e de outro, os resultados tecnológicos e econômicos da inovação. (INÁCIO e QUADROS, 2006, p. 26).
\end{abstract}

O IBI foi estruturado em dois conjuntos de indicadores, a saber: Indicador Agregado de Esforço (IAE) e Indicador Agregado de Resultado (IAR). Cada indicador congrega dois novos índices: Índice de Atividades Inovativas (IAI) e Índice de Recursos Humanos (IRH), compõem o IAE e o IAR é formado pelo Índice de Patentes (IP) e pelo Índice Impacto Econômico (II).

O cálculo do IBI, segundo, Furtado e Quadros (2006) segue a fórmula a seguir:

$$
\begin{aligned}
& I B I=\left(I A I \cdot P_{1}+I R H \cdot P_{2}\right) Q_{1}+\left(I P \cdot P_{3}+I I \cdot P_{4}\right) Q_{2} \\
& \text { Onde, } \\
& P_{1}+P_{2}=1 \quad P_{3}+P_{4}=1 \quad Q_{1}+Q_{2}=1
\end{aligned}
$$

A estrutura do IBI foi detalhada por Inácio e Quadros (2006) nas figuras que seguem. 
Figura 1 - Fórmula detalhada do IBI

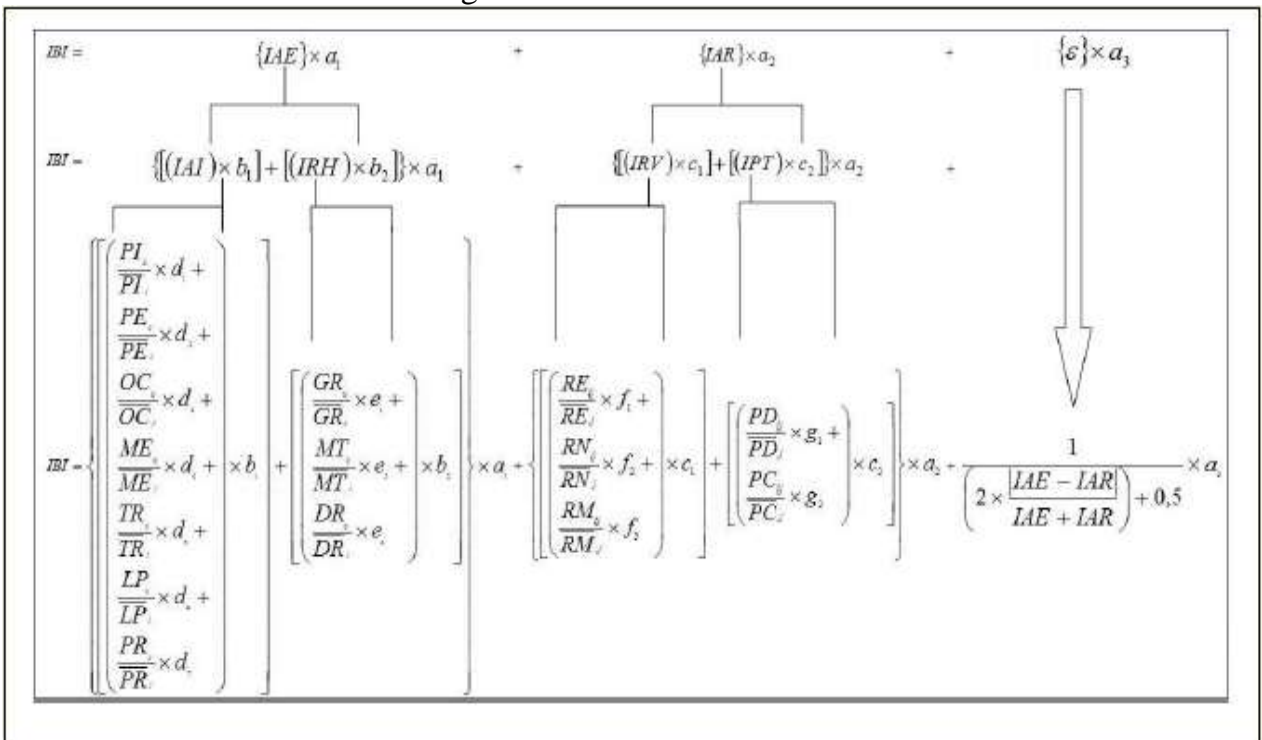

Fonte: Inácio e Quadros (2006, p. 27)

Figura 2 - Legenda do IBI

\begin{tabular}{|c|c|c|}
\hline \multicolumn{3}{|c|}{$\begin{array}{l}\text { 1. Quanto a nomenclatura: } \\
\text { As VARIAVEIS escritas em letras MAIUSCULAS são os diversos indicadores; } \\
\text { As variáveis escritas em letras minúsculas são os pesos atribuidos aos seus respectivos indicadores; } \\
V_{v} \text { representa o valor do indicador } V \text { para a empresa } i \text { do setor } j \text { e } V_{j} \text { a média da variável } V \text { do setor } j \text { da indústria de transformação, agrupada a } \\
\text { dois digitos da CNAE, calculada a partir dos dados da PINTEC (2003); }\end{array}$} \\
\hline 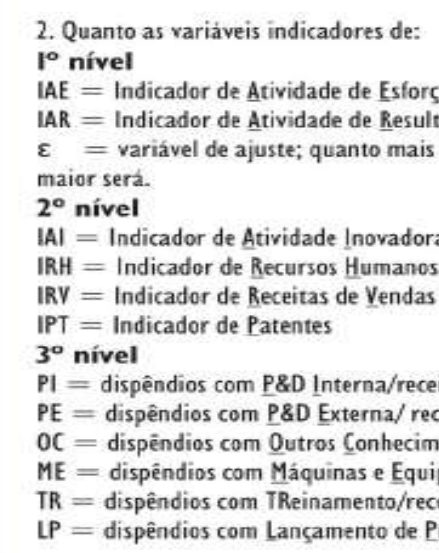 & $\begin{array}{l}\text { E e a IAR } \\
\text { s } \\
\text { quita liquida } \\
\text { quida }\end{array}$ & $\begin{array}{l}3^{\circ} \text { nivel } \\
\mathrm{PR}=\text { dispêndios com PRojeto Industrial/receita liquidação } \\
\mathrm{GR}=\text { total de GRaduados ocupados em P\&D/pessoal ocupado total; } \\
\mathrm{MT}=\text { total de Mes Ires ocupados em P\&D/pessoal ocupado total; } \\
\mathrm{DR}=\text { total de DoutoRes ocupados em P\&D/pessoal ocupado total; } \\
\mathrm{RE}=\text { Receita total de vendas (interna + externa) com produtos novos } \\
\text { para a Empresa/receita liquida; } \\
\mathrm{RN}=\text { Receita total de vendas (interna + externa) com produtos novos } \\
\text { para o mercado Nacional/receita liquida; } \\
\mathrm{RM}=\text { Receita total de vendas (interna + externa) com produtos novos } \\
\text { para o mercado Mundial/receita liquida; } \\
\mathrm{PD}=\text { total de Patentes Depositadas no periodo 2001-2003/ pessoal } \\
\text { ocupado total em } 2003 \\
\mathrm{PC}=\text { total de Patentes Concedidas no periodo 1994-2003/ pessoal } \\
\text { ocupado total em } 2003 \text {. }\end{array}$ \\
\hline $\begin{array}{l}\text { 3. Quanto as variáveis pesos de: } \\
1^{\circ} \text { nivel } \\
a_{1}+a_{2}+a_{3}=I(0,40 ; 0,40 \text { e } 0,20) \\
2^{\circ} \text { nivel } \\
b_{1}+b_{2}=I(0,75 ; 0,25) \\
c_{1}+c_{1}=I(0,60 ; 0,40)\end{array}$ & $\begin{array}{l}3^{\circ} \text { nivel } \\
d_{1}+d_{2}+ \\
e_{1}+e_{2}, e \\
f_{1}+f_{2}+f_{3} \\
g_{1}+g_{3}\end{array}$ & $\begin{array}{l}+d_{l}+d_{l}+d_{s}+d_{l}=1(0,30 ; 0,15 ; 0,10 ; 0,15 ; 0,05 ; 0,10 ; 0,15) \\
=1(0,15 ; 0,35 ; 0,50) \\
=1(0,10 ; 0,40 ; 0,60 \\
=I(0,50 ; 0,50)\end{array}$ \\
\hline
\end{tabular}

Fonte: Inácio e Quadros (2006, p. 27)

As ponderações realizadas para calcular o IBI, na parte inferior da Figura 2, não foram explicitadas, sugerindo uma decisão subjetiva sobre os mesmos.

O interesse dessa pesquisa é identificar e compreender o esforço em inovação tecnológica pela indústria nacional, a partir das três primeiras publicações da PINTEC, sem a preocupação no momento, de avaliar os impactos no resultado deste processo de inovação, tendo em vista, que a mensuração da influência da inovação no desempenho das empresas requer uma abordagem mais 
complexa, conforme aponta Santos (2009). Portanto, voltou-se o foco para o índices quem compõem o IAE (IAI + IRH).

A estrutura conceitual do IAI é coerente com o novo conceito de dispêndio com inovação que no passado era mensurado, apenas, pelos desembolsos na área de $\mathrm{P} \& \mathrm{D}$, sendo considerado atualmente como todos os gastos com inovação, inclusive aquisição de patentes, licenças, equipamentos e instalações (MOHNEN e DAGENAIS, 2002).

Ainda, alguns autores como Mansfield (1986), Levin et al. (1987), Cohen (1996) e Baldwin (1997) apud Baldwin, Hanel e Saboriun (2002), (ROS e LABEAGA, 2002), (MANGEMATIN e MANDRAN, 2002) (FEENY e ROGERS, 2003) asseguram que a mensuração, tão somente, do investimento em P\&D não capta o esforço inovador da firma como um todo.

Extrapolando o P\&D Interno Laursen e Salter (2006) defendem a necessidade do desenvolvimento de fontes externas de conhecimento como forma de diversificar os investimentos em inovação. Para estes pesquisadores a mobilidade que os "funcionários do conhecimento" possuem hoje aumenta o risco e o custo para as empresas investirem sozinhas no desenvolvimento de projetos. Pesa nessa estratégia a necessidade de operacionalizar uma rede de relacionamento capaz de absorver o conhecimento gerado em inovações de produtos e processos, porém atentando para os aspectos legais que envolvem tais transações (LAURSEN e SALTER, 2006).

Essa rede de relacionamento deve incluir diversos atores (fornecedores, universidades, centros de pesquisa, clientes, órgãos governamentais, sociedades de classe, entre outros) formando o chamado capital relacional, onde o estabelecimento de parcerias, alianças estratégicas e joint ventures podem fomentar e viabilizar o desenvolvimento de novos negócios ou aperfeiçoar os existentes (SAMPSON, 2007)

No que tange ao IRH, a proposta da Unicamp segue a orientação do Manual de Canberra ao considerar tanto o aspecto educacional quanto a ocupacional - função exercida e experiência nesta (DOMINGUES e FURTADO, 2006). A composição do índice é feita segundo os autores como:

Para fins de medição do IRH o número de pessoal de nível superior mobilizado em P\&D é normalizado pelo pessoal ocupado total de cada setor e ponderado de acordo com o nível de formação, de forma que o termo correspondente aos doutores, tem um peso maior, o que corresponde aos mestres um peso intermediário e o que corresponde aos graduados, o menor peso. Para formar o IRH, a soma dos recursos humanos relativos, alocados em tempo integral à $\mathrm{P} \& \mathrm{D}$, ponderada por nível de qualificação de cada empresa, é depois normalizada em relação à média setorial ponderada a dois dígitos. Com isso objetiva-se destacar as empresas com maiores e mais intensos esforços para inovar em relação ao seu respectivo setor, o que também se reflete pela melhor qualificação do pessoal mobilizado em P\&D. (DOMINGUES e FURTADO, 2006).

Andreassi e Sbragia (2002) identificaram que as empresas mais inovadoras, tinham as maiores quantidades de pessoas dedicadas no setor de $\mathrm{P} \& \mathrm{D}$, sendo que não necessariamente voltadas para novos projetos ou projetos finalizados, mas, também, na manutenção do 
conhecimento existente e acompanhamento das alterações ocorridas no mercado captados por indicadores como aquisição de tecnologias e engenharia não rotineira.

Santos e Popadiuk (2011) refoçam a importância fundamental do capital humano para o sistema de inovação da empresa e propõem um construto para mensurá-lo, com base em diferentes pesquisas realizadas nesta área (Inovação), reproduzido na Quadro 1.

\begin{tabular}{|l|l||}
\hline \multicolumn{2}{|c|}{ Quadro 1 - Modelo de Construto para o Capital Humano } \\
\hline Número de engenào & \multicolumn{1}{c|}{ Caracteristicas } \\
\hline $\begin{array}{l}\text { Número de administradores } \\
\text { (gerentes) }\end{array}$ & $\begin{array}{l}\text { Tempo de experiência na ati- } \\
\text { vidade }\end{array}$ \\
\hline $\begin{array}{l}\text { Número de doutores, mestres, } \\
\text { graduados, técnicos e suporte } \\
\text { dedicados a P\&D }\end{array}$ & $\begin{array}{l}\text { Exposiçào ao relacionalónento } \\
\text { externo com clientes e forne- } \\
\text { cedores }\end{array}$ \\
\hline & $\begin{array}{l}\text { Dispêndio com salários, en- } \\
\text { cargos, beneficios e prêmios } \\
\text { dos funcionários envolvidos } \\
\text { com P\&D }\end{array}$ \\
\hline
\end{tabular}

Fonte: Adaptado de Santos e Popadiuk (2011, p. 111)

No entanto, o formulário aplicado pela PINTEC/IBGE não traz as variações propostas por Santos e Popadiuk (2011) no que remete ao número de engenheiros e administradores, apenas o número de pessoal dedicado a $\mathrm{P} \& \mathrm{D}$, tal qual é calculado pelo Indice de Recursos Humanos do IBI.

Em razão da abordagem teórica providenciada, constata-se não apenas os fundamentos da inovação para as empresas e seus reflexos nestas, mas a estrturura que assenta a capacidade de inovar das empresas baseada no modelo proposto para o IBI; com efeito, pode-se, sem prejuízo espúrio, identificar o perfil da inovação da indústria brasileira com base nos índices que compõem o Indicador Agregado de Esforço.

\section{Procedimentos metodológicos}

A pesquisa tem natureza metodológica exploratória, tendo em vista, que não foram alcançados trabalhos com estas características no Brasil, a partir, dos dados da PINTEC; o próprio IBI não acompanhou as duas últimas edições da pesquisa (2005 e 2008) empreendida pelo IBGE. Desta forma, este estudo exploratório poderá sinalizar novos horizontes de pesquisa neste eixo do conhecimento e ainda permitir uma perspectiva do esforço em inovação na indústria brasileira na primeira metade da última década.

A abordagem é quantitativa com interesse descritivo, portanto, serão fornecidos dados absolutos da amostra e medidas convencionais de posição (Média) e dispersão (Desvio Padrão), 
cujo entendimento e metodologia de cálculo são pacíficos.

Os dados foram coletados nas instalações do IBGE em 2008 e 2009 sendo consolidados em planilhas eletrônicas que auxiliaram no desenvolvimento das tabelas. Em função do interesse em verificar a evolução da rentabilidade da indústria no período, providenciou-se uma merger da base da PINTEC com uma base do autor, proveniente da Gazeta Mercantil. Esse procedimento foi necessário para ampliar o entendimento do esforço de inovação das empresas, tendo em vista, que o dado agregado da inovação no Brasil, per si, pode ser visualizado no domínio da PINTEC na Internet (www.pintec.ibge.gov.br).

Importa apresentar a metodologia da PINTEC para fins de validação do seu uso. A estrutura da pesquisa promovida pelo IBGE tem por referência conceitual o Manual de Oslo e segue o modelo aplicado pela Oficina Estatística da Comunidade Européia - EUROSTAT, a terceira versão da Community Innovation Survey - CIS 1998 - 2000 (IBGE, 2007, p. 13). A pesquisa tem por objetivo construir indicadores setoriais, nacionais e regionais do processo de inovação das firmas instaladas no Brasil, que atendessem aos seguintes requisitos (IBGE, 2007, p. 14):

- Estar em situação ativa no Cadastro Central de Empresas - CEMPRE, do IBGE;

- Ter atividade principal a indústria extrativista ou de transformação;

- Ter dez ou mais pessoas ocupadas em 31 de dezembro do ano de referência.

A amostra trabalhada envolveu em 2000, 2003 e 2005 um total de 1.712, 1.586 e 1.562 empresas respectivamente. Todavia, vale ressaltar, que nem todas as empresas pesquisadas, dedicaram ou mesmo possuem esforço inovador em todos os índices trabalhados, fato que poderá ser observado na análise dos resultados, contudo, ressalta-se que todas as empresas declararam ter realizado algum tipo de inovação no período correspondente.

Ressalva-se, que houve uma mudança na forma de preenchimento do formulário da PINTEC, após a edição de 2000. Na primeira pesquisa não havia campos nulos, assim, quando a empresa não informava algum dado, o sistema pontuava automaticamente como zero " 0 ", desta forma, não se sabia se o valor era de fato zero, ou se a empresa, por algum motivo não informou.

Nas pesquisas seguintes (2003 e 2005) esse fato não ocorreu, pois as empresas foram orientadas a informar o valor zero se realmente o investimento foi nulo, ou poderiam deixar em branco, na hipótese de não desejarem informar o dado. Essa diferença poderá ser notada nos resultados pelas variações no número de respondentes.

A estratificação dos setores com o percentual de empresas, não será apresentado, em razão do compromisso de confidencialidade de informação junto ao IBGE, tendo em vista, que alguns setores apresentam pouquíssimas empresas, permitindo inferências quanto a sua razão social. 
As variáveis utilizadas em consonância ao referencial teórico desenvolvido encontram-se na Quadro 2, bem como, a forma de cálculo.

Quadro 2 - Descrição das variáveis

\begin{tabular}{|l|l|}
\hline \multicolumn{1}{|c|}{ Título } & \multicolumn{1}{c|}{ Cálculo } \\
\hline Doutores & Quantidade de doutores dedicados P\&D em relação ao número de funcionários. \\
\hline Mestres & Quantidade de mestres dedicados a P\&D em relação ao número de funcionários. \\
\hline Graduados & Quantidade de graduados dedicados a P\&D em relação ao número de funcionários \\
\hline Técnicos & Quantidade de técnicos dedicados a P\&D em relação ao número de funcionários. \\
\hline Total_hum_ped & Total de pessoas dedicadas a P\&D em relação ao número de funcionários. \\
\hline Treinamento & Total dos dispêndios em treinamento dividido pela receita da firma. \\
\hline P\&D Interno & Total dos dispêndios em P\&D interno dividido pela receita da firma. \\
\hline Aquisição de Máquinas & Total dos dispêndios em aquisição de máquinas pela receita da firma. \\
\hline $\begin{array}{l}\text { Introdução de Inovação } \\
\text { Tecnológica }\end{array}$ & $\begin{array}{l}\text { Total dos dispêndios em introdução de inovações tecnológicas dividido pela receita } \\
\text { da firma. }\end{array}$ \\
\hline P\&D Externo & Total dos dispêndios em P\&D externo dividido pela receita da firma. \\
\hline Conhecimento Externo & $\begin{array}{l}\text { Total dos dispêndios na aquisição de conhecimento externo dividido pela receita da } \\
\text { firma. }\end{array}$ \\
\hline
\end{tabular}

Fonte: Autoria própria (2012)

O procedimento adotado para relativizar os resultados brutos da PINTEC deve-se a necessidade de considerar as empresas pelo seu tamanho, seja em número de funcionários ou valor da receita total, desta feita, tem-se uma "padronização" das empresas.

As variáveis de rentabilidade utilizadas, a partir, dos dados financeiros conseguidos junto a Gazeta Mercantil foram às métricas tradicionais de ROE (Return on equity), ROA (Return on assets) e ROS (Return on sales).

$$
\begin{array}{r}
R O E=\frac{\text { Lucro_Líquido }}{\text { Patrimônio_Líquido }} \\
R O A=\frac{\text { Lucro_Líquido }}{\text { Ativo_Total }} \\
R O S=\frac{\text { Lucro_Líquido }}{\text { Receita_Líquida }}
\end{array}
$$

Em função da característica e interesse da pesquisa, os resultados correspondem ao perfil da indústria na sua totalidade, condicionado a amostra não probabilística. Portanto, não se podem inferir tais resultados para empresas específicas ou setores, que merecem estudos adicionais, além disso, em razão do limite temporal as informações devem ser consideradas como fatos para o período definido. Essas informações devem ser consideradas, quando os resultados aqui apresentados forem comparados com outras pesquisas ou unidades de análise.

Destaca-se, ainda, que não se procedeu com nenhuma modificação na amostra, em especial, na exclusão de "outliers", em virtude, de não se trabalhar com metodologias de análise multivariada. De forma, que como se trata de uma amostra ampla, os resultados mais distantes tornam-se, em certa medida, "normalizados" à média. Essa decisão foi tomada, no sentido, de evitar 
um procedimento subjetivo sobre os valores correspondentes aos limites inferiores e superiores, com prejuízo à amostra.

\section{Análise dos resultados}

A proposta para análise dos resultados, no que tange a apresentação das informações ocorrerá de forma analítica por pesquisa (2000, 2003 e 2005), sendo que a avaliação longitudinal dar-se-á de forma cumulativa no decorrer da seção.

A PINTEC edição 2000 alcançou 72.005 empresas que empregavam à época 4.959.623 funcionários, todavia, segundo dados publicados, apenas 31,52\% (22.698) dessas empresas afirmaram ter realizado algum tipo de inovação (em produto ou processo; incremental ou radical) (PINTEC, 2002).

Após a merger com a base da Gazeta Mercantil, constituiu-se uma amostra mais restrita com 1.712 empresas que reportaram no formulário alguma informação quanto aos funcionários dedicados à pesquisa e desenvolvimento, cujos resultados em relação ao efetivo, encontram-se na Tabela 1.

Tabela 1 - Variáveis de Recursos Humanos (PINTEC 2000).

\begin{tabular}{cccccc}
\hline Variáveis & $\mathbf{N}$ & Mínimo & Máximo & Média & Desvio Padrão \\
\hline Graduados & 1712 &, 00 &, 36 &, 0050 &, 02132 \\
Técnidos & 1712 &, 00 &, 35 &, 0038 &, 01553 \\
Total_hum_ped & 1712 &, 00 &, 53 &, 0102 &, 03872 \\
Mestres & 1712 &, 00 &, 11 &, 0007 &, 00547 \\
Doutores & 1712 &, 00 &, 52 &, 0007 &, 01411 \\
\hline
\end{tabular}

Fonte: Autoria própria (2012)

Verifica-se que a média de funcionários dedicados à P\&D no Brasil representa $1 \%$ do total de funcionários das empresas, sendo que aproximadamente $90 \%$ desse pequeno número foi formado por profissionais de nível técnico e graduação, de forma que a representatividade de mestres e doutores dedicados à pesquisa é bem pequeno.

Para se ter uma dimensão do que esse número representa, o total de funcionários em P\&D das 72.005 empresas era de 41.467 pessoas, o que entrega uma média de $0,8 \%$ do efetivo total, próximo do resultado alcançado na amostra da Tabela 1; em outra medida, para cada duas empresas, uma possuía uma única pessoa dedicada em P\&D. Se considerar mestres e doutores, uma a cada 7 empresas apresentaria, ao menos, um mestre ou doutor, dedicado à pesquisa e desenvolvimento.

Essa evidência corrobora a informação de Sbragia (2006) quando o autor cita a baixa participação de cientistas e engenheiros brasileiros em pesquisa dentro das empresas.

Além disso, a amplitude entre o mínimo e o máximo é alta, conforme retrata os desvios padrões das variáveis. Esse resultado é influenciado tanto pela disparidade tecnológica da indústria 
brasileira, onde existem alguns "oásis" de inovação quanto pela heterogeneidade da amostra que abrange empresas a partir de 10 funcionários e de todos os setores industriais, o que enviesa a informação absoluta.

No que diz respeito às atividades inovativas, os dados agregados da PINTEC apontam para um investimento total da ordem de $\mathrm{R} \$ 22,343$ bilhões (em valores à época), sendo que 19.165 empresas declararam ter realizado algum investimento em inovação (PINTEC, 2002). Esses resultados asseguram uma média de investimento por empresa de $\mathrm{R} \$ 1,165$ milhão.

A Tabela 2 apresenta os resultados relativizados pelas receitas das empresas selecionadas para a amostra dessa pesquisa.

Tabela 2 - Variáveis das Atividades Inovativas (PINTEC, 2000).

\begin{tabular}{cccccc}
\hline Variáveis & $\mathbf{N}$ & Mínimo & Máximo & Média & Desvio Padrão \\
\hline Treinamento & 1712 &, 00 & 33,33 & 0,0217 & 0,8053 \\
P\&D Interno & 1712 &, 00 & 2166,67 & 1,2851 & 52,3346 \\
Aquisição de Máquinas & 1712 &, 00 & 545,33 & 0,5083 & 13,8920 \\
P\&D Externo & 1712 &, 00 & 1,43 & 0,0025 & 0,0383 \\
Conhecimento Externo & 1712 &, 00 & 0,98 & 0,0029 & 0,0332 \\
Introdução de Inovação & 1712 &, 00 & 333,33 & 0,1981 & 8,0513 \\
Tecnológica & & & &
\end{tabular}

Fonte: Autoria própria (2012)

Pode-se perceber num primeiro momento, que o P\&D Interno foi, na média, o item com maior investimento pelas empresas brasileiras, seguido pela Aquisição de Máquinas, todavia, observa-se que o valor máximo influenciou a média final, como demonstra o desvio padrão. Esse resultado sinaliza novamente a disparidade dos investimentos em P\&D no país, bem como o comportamento de algumas empresas em específico, que não foi possível retirar da amostra pelas razões explicitadas na seção anterior.

Apesar dessa distorção, se proceder com a redução de um desvio padrão na média, poder-seá observar uma redução significativa no valor médio de investimento nas duas variáveis. Além disso, como o limite mínimo é 0,00 , não se tem uma distribuição normal, o que concentra os dados próximos dessa nova média.

Outra variável que apresentou elevada amplitude entre os extremos foi a Introdução da Inovação tecnológica que por certo puxou a média para cima, tendo em vista o mínimo em 0,00 e a média em 0,1981.

As outras três variáveis permitem uma análise mais coerente, em razão do menor desvio padrão, de maneira, que os investimentos em Treinamento são mais frequentes e valorizados à Aquisição de Conhecimento Externo e o P\&D Externo. Por outro modo, pode-se dizer que houve um esforço maior no desenvolvimento do conhecimento interno das empresas a busca por fontes externas. 
As variáveis de rentabilidade da indústria, como não poderiam ser diferentes, foram dispersas, todavia, sinalizam de forma agregada o crescimento do país no período. Nesta etapa, não foi possível alcançar as 1.712 empresas, pois 64 empresas, não apresentaram a informação quanto o lucro líquido do período. De toda forma, o resultado agregado não se torna inválido, conforme, a Tabela 3.

Tabela 3 - Variáveis de Rentabilidade (Ano base 2000).

\begin{tabular}{cccccc}
\hline Variáveis & N & Mínimo & Máximo & Média & Desvio Padrão \\
\hline ROA & 1648 & $-6,42$ & 1,88 &, 0067 &, 27046 \\
ROE & 1648 & $-281,07$ & 361,83 &, 0175 & 11,42519 \\
ROS & 1648 & $-13,52$ & 63,78 &, 0218 & 1,83256 \\
\hline
\end{tabular}

Fonte: Autoria própria (2012)

A média do setor foi positiva em todos os anos, fruto do resultado obtido no período, contudo, a rentabilidade da indústria de transformação é baixa, onde na média é inferior ao custo de oportunidade do país, de $6 \%$ a.a. se considerada a taxa de remuneração da poupança nacional.

No conjunto dos resultados para o ano de 2000, pode-se constatar a falta de pesquisadores nas empresas e a predominância de investimento em fontes internas à inovação, exceção à Aquisição de Máquinas. Ressalta-se, ainda, que a inserção das empresas em redes de relacionamento para o desenvolvimento de novas fontes de conhecimento e inovação é quase nulo.

A PINTEC edição 2003, publicada em 2005, contabilizou 84.262 empresas que empregavam 5.353.909 funcionários; destas empresas, 33,27\% conseguiram implementar alguma inovação em produto ou processo; percentual muito próximo do realizado em 2000 (PINTEC, 2005).

A Tabela 4 apresenta os resultados referentes ao pessoal dedicado à pesquisa e desenvolvimento.

Tabela 4 - Variáveis de Recursos Humanos (PINTEC, 2003).

\begin{tabular}{lrrrrr}
\hline Variáveis & N & Mínimo & Máximo & \multicolumn{1}{c}{ Média } & Desvio Padrão \\
\hline Graduados & 613 &, 00 &, 57 &, 0207 &, 05739 \\
Técnicos & 613 &, 00 &, 70 &, 0143 &, 05034 \\
Total_hum_ped & 613 &, 00 & 1,00 &, 0409 &, 09967 \\
Mestres & 613 &, 00 &, 15 &, 0024 &, 00926 \\
Doutores & 613 &, 00 &, 11 &, 0009 &, 00610 \\
\hline Fonte: Autoria própria (2012) & & & & &
\end{tabular}

Fonte: Autoria própria (2012)

A diferença metodológica no preenchimento da PINTEC, a partir desta pesquisa pode ser observada na Tabela 4, pois apesar da amostra trabalhada de 1.586 empresas em 2003 com algum tipo de inovação tecnológica, apenas 613 empresas destas preencheram o quantitativo de pessoas em Pesquisa e Desenvolvimento, o que torna mais real o número para as empresas com P\&D, porém impede uma análise comparativa em número absoluto com os dados de 2000.

Observa-se que, agora, 4,09\% do quantitativo dos funcionários estavam dedicados à P\&D, onde continua a preponderância de graduados e técnicos, tal como em 2000. A participação de 
mestres é bem maior que doutores, porém muito inferior aos graduados. Em termos relativos, dentro da participação deste grupo em P\&D, observa-se, que em 2003, mantém-se a representatividade de graduados e técnicos no $\mathrm{P} \& \mathrm{D}$ das empresas.

Tomando, como base os dados agregados informados pela PINTEC edição 2003, 21.795 pessoas estavam dedicadas ao $\mathrm{P} \& \mathrm{D}$ nas empresas nesta pesquisa, o que representa $0,4 \%$ do efetivo total, tornando, os resultados em média inferiores aos identificados na pesquisa de 2000 (PINTEC, 2005). Além disso, percebe-se a elástica diferença entre o quantitativo de funcionários dedicados em $\mathrm{P} \& \mathrm{D}$ na amostra contra a média da indústria, o que lastreia a afirmação referente à concentração de pesquisa em poucas empresas.

No tocante ao esforço em atividades inovativas no ano de 2003 , a pesquisa contabilizou no agregado 20.599 empresas com investimentos em inovação, totalizando um valor superior a $\mathrm{R} \$$ 23,419 bilhões, ou seja, um valor médio por empresa de R \$ 1,136 milhão (PINTEC, 2005). Este valor absoluto é muito semelhante ao valor de 2000, porém, quando considerada a inflação acumulada do período (29,5\%), tem-se um valor deflacionado de investimento médio por empresa em R $\$ 800$ mil, ou seja, houve uma redução de 31,27\% de recursos destinado à inovação entre a primeira e segunda edição da PINTEC.

A Tabela 5 traz os resultados para a amostra trabalhada junto os microdados no IBGE.

Tabela 5 - Variáveis das Atividades Inovativas (PINTEC, 2003).

\begin{tabular}{lrrrrr}
\hline Variáveis & \multicolumn{1}{c}{ N } & Mínimo & Máximo & Média & Desvio Padrão \\
\hline Treinamento & 773 &, 00 &, 27 &, 0024 &, 01388 \\
P\&D Interno & 636 &, 00 & 10,23 &, 0578 &, 50973 \\
Aquisição de Máquinas & 835 &, 00 & 140,66 &, 2176 & 4,87110 \\
P\&D Externo & 1585 &, 00 &, 55 &, 0018 &, 02277 \\
Conhecimento Externo & 1582 &, 00 &, 45 &, 0016 &, 01538 \\
Introdução de Inovação & 445 &, 00 & 19,18 &, 0710 &, 94232 \\
Tecnológica & & & & &
\end{tabular}

Em função da alteração no preenchimento do formulário, os dados consolidados tornaram-se mais realistas, também, para as atividades inovativas, conforme a distribuição dos valores dentro dos limites máximos e mínimos. Apesar disso, observa-se que todas as variáveis perderam representatividade na participação das receitas, quando comparado com a primeira edição da pesquisa.

A variável Aquisição de Máquinas foi a mais preponderante entre as atividades inovativas, mesmo considerando o alto desvio padrão. A variável P\&D Interno que na pesquisa de 2000 foi a principal, assume uma posição mais modesta o que, em certa medida, coaduna, com a baixa participação de pesquisadores em P\&D na indústria nacional. 
A variável Introdução da Inovação Tecnológica, em participação na receita, assume a segunda posição, o que demonstra a preocupação do industriário em viabilizar comercialmente a inovação.

O investimento no desenvolvimento de fontes de Conhecimento e P\&D externos continuam com uma representatividade muito pequena, quando comparada com o P\&D Interno, por exemplo. Situação semelhante ao evidenciado na edição de 2000.

A variável "Treinamento" assume a quarta colocação no esforço inovador das empresas nesta edição. Desta forma, observa-se que o esforço inovador das empresas no período continua na atualização de suas estruturas produtivas, através da Aquisição de Máquinas, cujo conteúdo tecnológico é desenvolvido fora da firma.

A Tabela 6 apresenta a rentabilidade de 86,32\% da amostra trabalhada no ano de 2003.

Tabela 6 - Variáveis de Rentabilidade (Ano base 2003)

\begin{tabular}{lccccc}
\hline Variável & N & Mínimo & Máximo & Média & Desvio Padrão \\
\hline ROA & 1369 & $-2,65$ & 2,69 &, 0409 &, 21704 \\
ROE & 1369 & $-22,04$ & 129,98 &, 1719 & 3,83419 \\
ROS & 1369 & $-5,25$ & 74,81 &, 0822 & 2,06389 \\
\hline
\end{tabular}

Fonte: Autoria própria (2012)

Verifica-se, que no agregado, o nível de rentabilidade aumentou significativamente nas empresas, onde se destaca o retorno para os empresários (ROE) superior a 17\%, contra, 1,75\% apurado para a base de 2000 .

Interessa, analisar que os investimentos em inovação não acompanharam o crescimento da rentabilidade das empresas nacionais, onde pondera-se aqui, que a inovação não é de fato uma estratégia de longo prazo da indústria brasileira.

Constata-se que a mudança na forma de preenchimento da PINTEC permitiu uma análise mais acurada dos dados, tendo em vista a menor dispersão apresentada por estes; no entanto, os resultados, apontados, continuam a sinalizar para uma baixa participação de pesquisadores ou profissionais dedicados à inovação nas empresas, bem como, uma redução na participação de recursos destinados à inovação tanto nos valores agregados, como nos percentuais relativizados pela receita.

A pesquisa referente ao período de 2005, publicada em 2007, manteve as alterações no procedimento de preenchimento do ano de 2003, o que permitiu uma análise comparativa mais segura entre as duas últimas pesquisas. Na edição de 2005 a PINTEC conseguiu atingir 95.301 empresas, número recorde, em relação às anteriores; conquanto destas, 32.796 afirmaram ter implementado alguma inovação, ou seja, 34,41\%, número superior às pesquisas anteriores, porém com modesto crescimento, com efeito, tem-se para o período uma média de $33 \%$ das indústrias com algum tipo de inovação implementada, em cada período (PINTEC, 2007). 
Destas que responderam positivamente a implementação de inovação, 1.562 constituíram a amostra trabalhada, mantendo assim, uma razoável representatividade da indústria brasileira.

A Tabela 7 apresenta os resultados das variáveis de recursos humanos dedicadas à inovação na amostra analisada.

Tabela 7 - Variáveis de Recursos Humanos (PINTEC, 2005)

\begin{tabular}{lccccc}
\hline Variáveis & $\mathbf{N}$ & Mínimo & Máximo & Média & Desvio Padrão \\
\hline Graduados & 573 &, 00 & 1,33 &, 0217 &, 07518 \\
Técnicos & 573 &, 00 &, 21 &, 0111 &, 02614 \\
Total_hum_ped & 573 &, 00 & 1,67 &, 0394 &, 10111 \\
Mestres & 573 &, 00 &, 33 &, 0029 &, 01581 \\
Doutores & 573 &, 00 &, 19 &, 0013 &, 00943 \\
\hline
\end{tabular}

Fonte: Autoria própria (2012)

Dentro da amostra pesquisada há uma redução de empresas respondentes quanto ao pessoal dedicado à inovação, contudo, os dados continuam mais robustos, assim como em 2003. No agregado dedicado em $P \& D$, verifica-se uma ligeira queda no percentual de 4,08\% para 3,94\%, que apesar de pequeno é frustrante face o baixo quantitativo de pessoas dedicadas à inovação. Contudo, observa-se uma maior participação de mestres e doutores na composição das equipes de inovação, em que pese, o maior peso, ainda, de graduados e técnicos em pesquisa.

O agregado da PINTEC edição 2005, apontou um total de 49.354 pessoas dedicadas à P\&D frente o universo de 6.409.876 trabalhadores, o que representa 0,76\% do efetivo dedicado a pesquisa e inovação. Ressalta-se que esse resultado é bem superior ao de 2003 (0,4\%), porém, ainda inferior ao identificado em 2000.

Desta forma, pode-se afirmar que não houve melhoria no quantitativo de pessoas dedicadas à $\mathrm{P} \& \mathrm{D}$ na indústria brasileira, que apesar de aumentar de forma absoluta, o percentual de trabalhadores com características para gerar inovação é incipiente e ainda foi reduzido, quando relativizado pela quantidade de novos trabalhadores nos setores industriais. Essa situação compromete a competitividade da indústria nacional no cenário globalizado e desperta para a necessidade de investimentos intensivos na formação e valorização de pesquisadores dentro das empresas.

$\mathrm{O}$ investimento agregado em atividades inovativas alcançou o montante de $\mathrm{R} \$ 41,289$ bilhões para um total de 21.966 empresas (PINTEC, 2007), o que representa um investimento médio por empresa de R\$1,879 milhão, de forma, que quando deflacionado $(42,79 \%)$ para o ano 2000 tem-se um valor médio real de $\mathrm{R} \$ 804$ mil por empresa.

Este resultado é muito próximo ao registrado em 2003 e por consequência inferior ao ano base 2000. Portanto, não houve crescimento real no valor dos investimentos em atividades inovativas no período analisado. 
A Tabela 8 traz os resultados consolidados para as variáveis que compõem as atividades inovativas para a amostra trabalhada junto a base de microdados no IBGE.

Tabela 8 - Variáveis das Atividades Inovativas (PINTEC, 2005).

\begin{tabular}{lrrrrr}
\hline Variáveis & \multicolumn{1}{c}{ N } & Mínimo & Máximo & \multicolumn{1}{c}{ Média } & Desvio Padrão \\
\hline Treinamento & 910 &, 00 & 3,00 &, 0042 &, 09955 \\
P\&D Interno & 648 &, 00 & 8,12 &, 0401 &, 43451 \\
Aquisição de Máquinas & 972 &, 00 & 78,41 &, 1872 & 2,90846 \\
P\&D Externo & 1562 &, 00 &, 05 &, 0005 &, 00304 \\
Conhecimento Externo & 1561 &, 00 &, 16 &, 0009 &, 00725 \\
Introdução Inovação & 557 &, 00 & 13,84 &, 0302 &, 58634 \\
Tecnológica & & & & &
\end{tabular}

Assim, como em 2003 a pesquisa realizada em 2005 apresentou resultados menos dispersos, expressos pelo desvio padrão, contudo, em 2005, observa-se uma redução média dos investimentos em atividades inovativas em todas as variáveis, exceção a Treinamento, que mesmo assim, possui pequena participação (Média de $0,42 \%$ da receita).

As maiores quedas absolutas ocorreram na Aquisição de Máquinas e na Introdução de Inovação Tecnológica, sendo, que os investimentos em P\&D Externo e Conhecimento Externo, passam a ser insignificantes.

A Tabela 9 apresenta a rentabilidade da indústria no ano de 2005, onde a dispersão se mantém, pelos fatores já identificados, porém, observa-se uma queda brusca na rentabilidade do setor, que na média foi negativa.

Tabela 9 - Variáveis de Rentabilidade (Ano base 2005).

\begin{tabular}{lcrrrr}
\hline Variáveis & N & \multicolumn{1}{c}{ Mínimo } & \multicolumn{1}{c}{ Máximo } & \multicolumn{1}{c}{ Média } & Desvio Padrão \\
\hline ROA & 1303 & $-149,50$ & 1,34 &,- 0736 & 4,14628 \\
ROE & 1303 & $-4196,08$ & 644,61 & $-3,5845$ & 126,24970 \\
ROS & 1303 & $-27,10$ & 22,69 &,- 0198 & 1,15539 \\
\hline
\end{tabular}

Fonte: Autoria própria (2012)

Essa queda pode estar associada ao fraco crescimento econômico do período (Aumento do PIB em 2,5\%), bem como a dificuldade da indústria nacional em competir no exterior quando a taxa de câmbio não é favorável.

Assim, no compromisso de consolidar os objetivos propostos por esta pesquisa, pode-se afirmar que:

a) Existe uma grande heterogeneidade no comportamento da indústria brasileira, quando se refere ao esforço em gerar inovação. Essa diferença foi identificada pelo alto desvio padrão nas variáveis em todos os anos. Infere-se que essa diversidade sofre influência, das diferenças tecnológicas existentes entre os diferentes setores industriais, mas principalmente, pela concentração de esforços em inovação em algumas empresas brasileiras; 
b) A principal variável que sustenta a inovação no Brasil é a Aquisição de Máquinas, o que explica um perfil voltado para modernização e acompanhamento tecnológico. Essa evidência reforça os trabalhos de Sbragia (2006) e Arruda, Vemulm e Hollanda (2006). Por outro modo, assevera-se que o perfil da indústria brasileira não é a busca pela vanguarda tecnológica, frente os baixos e declinantes investimentos em P\&D Interno e desenvolvimento de Conhecimento e P\&D Externo;

c) Os investimentos em inovação, em especial, àquelas atividades que podem proporcionar mudanças radicais não são contempladas pela indústria nacional como um todo, em razão da baixa representatividade de recursos destinados a P\&D Interno e Externo. Observa-se, que para as dimensões da indústria brasileira o processo inovador, ainda é, algo distante;

d) Há, de fato, baixa participação de profissionais com perfil orientado à pesquisa (mestres e doutores) nas empresas brasileiras. Ainda, os funcionários dedicados à "pensar" as inovações nas empresas representam menos de $1 \%$ da força de trabalho das indústrias brasileiras. Essa evidência confirma as pressuposições 2 e 3 .

\section{Conclusão}

O interesse dessa pesquisa foi identificar e compreender, dentro das limitações metodológicas, o perfil da inovação na indústria brasileira. Para tanto, diagnosticou-se dentro da literatura, em especial, nos trabalhos de Schumpeter e no pensamento evolucionário, que o processo de crescimento econômico, seja do país ou da firma, passa, necessariamente por investimentos no fomento de inovação, em especial, em P\&D, seja dentro ou fora das empresas. Não obstante, observou-se, também, que a inovação só tem sentido, na linguagem econômica, se esta for aplicada, isto é, a inovação precisa gerar novos produtos ou processos de produtos que proporcionem rents de inovação.

A estrutura utilizada para dimensionar o esforço em inovação tomou como base o Índice Brasil de Inovação, que conforme observado possui lastro na literatura pesquisada. A partir deste modelo utilizou-se os índices que compõem o indicador agregado de esforço (IAE), sendo eles: Índice de Recursos Humanos e o Índice de Atividades Inovativas.

Lançou-se mão dos dados secundários provenientes da PINTEC (2000, 2003 e 2005) onde os resultados agregados constituíram-se pano de fundo para os resultados de uma amostra proveniente de uma merger com uma base de dados financeiros de empresas nacionais provenientes da Gazeta Mercantil.

Os resultados da pesquisa sinalizam que o perfil da indústria brasileira é voltado para o acompanhamento tecnológico, tendo em vista: preponderância de investimento em Aquisição de Máquinas; modestos investimentos em P\&D dentro das empresas, sendo que o perfil dos 
funcionários dedicados à esta atividade não é voltado para pesquisa e irrelevância nos investimentos em fontes externas de Conhecimento e Pesquisa.

Identificou-se, também, que o investimento médio por empresa caiu no curso do tempo, bem como a participação de profissionais dedicados à $\mathrm{P} \& \mathrm{D}$ quando relativizado pelo efetivo total. Essas evidências confirmam a dificuldade da economia brasileira em ganhar uma dinâmica própria e mais homogênea.

Diante dessas constatações, alçam-se como investigações futuras, questões relativas ao comportamento não da indústria como um todo, mas dos setores, discussões que envolvam a rentabilidade de empresas com mais e menos inovação, políticas de financiamento a inovação, novas estruturas de inovação, em especial, aquelas que envolvem redes de inovação. Aumento nas pesquisas do tipo "estudo de caso", a fim, de permitir um melhor conhecimento dos processos e resultados da inovação dentro de um negócio, entre outros.

Importa, que novas pesquisas nessa temática sejam levantadas, pois o cenário é de dês(industrialização) e aquelas que se mantém, continuam com o perfil de replicador de tecnologia, ou de plataforma de produção a mercê de políticas fiscais pontuais e da taxa de câmbio. Por isso, tem-se há necessidade de uma política industrial que supere fragilidade tecnológica onde se assenta o tecido industrial brasileiro.

\begin{abstract}
This study examines the behavior of the efforts of the Brazilian industry in innovation from the results of PINTEC / IBGE in 2000, 2003 and 2005. Was taken as the reference model of the Innovation Index Brazil (Furtado and TABLES, 2006) regarding the aggregate indicator of effort (IAE). From a descriptive analysis of aggregate information PINTEC and selected an average sample of 1,620 companies, it was found that the profile of innovation in Brazilian industry is focused on monitoring / technological modernization, in order that the main investment innovation occurs in Acquisition of machinery, so that resources allocated to internal R \& D are modest and those oriented to the development of Knowledge and Research outside the company are negligible. Nevertheless, the participation of teachers and doctors in research in the national industry is less than $0.5 \%$ of the total workforce. Thus, it appears that the profile of the domestic industry is not geared to innovation, but this monitoring.
\end{abstract}

Key-words: innovation; Brazilian industry; PINTEC/IBGE.

\title{
Referências
}

ANDREASSI, T. Estudo das relações entre indicadores de P\&D e indicadores de resultado empresarial em empresas brasileiras. 1999. 213f. Tese (Doutorado em Administração), Faculdade de Economia e Administração, Universidade de São Paulo, São Paulo, 1999.

ANDREASSI, T.; SGRAGIA, R. Relações entre indicadores de P\&D e de resultado empresarial. Revista da Administração da USP - RAUSP, v. 35, n. 1, p. 63-71, 2002.

ARRUDA, M. F. M. A indústria e o desenvolvimento tecnológico nacional. Ciência e Tecnologia: alicerces do desenvolvimento. São Paulo: CNPq/COBRAMM, p. 23-44, 1994. 
ARRUDA, M.; VERMULM, R.; HOLLANDA, S. Inovação tecnológica no Brasil. A indústria em busca da competitividade global. São Paulo: Anpei, 2006.

BALDWIN, J.; HANEL, P.; SABORIUN, D. Determinants of innovative activity in canadian manufacturing firms. In. KLEINKNECHT, A.; MOHNEN, P. Innovation and firm performance. Econometric explorations of survey data. New York: Palgrave, 2002.

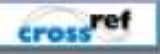

CONCEIÇÃO, O. A. C. A relação entre processo de crescimento econômico, mudança e instituições na abordagem institucionalista. Ensaios FEE, Porto Alegre, v. 23, especial, p. 603-620, 2002.

DOMINGUES, S. A.; FURTADO, A. Recursos Humanos em P\&D. Uniemp Inovação, Campinas, v. 2, n. 4, p. 26-27, 2006.

DOSI, G. Innovation, organization and economic dynamics. Selected essays. Cheltenham: Edward Elgar Publishing, 2000 .

FEENY, S.; ROGERS, M. Innovation and performance: benchmarking australian firms. The Australian Economic Review. Melbourne, v. 36, n. 3, p. 253-264, 2003.

FURTADO, A.; QUADROS, R. Construindo o IBI. Uniemp Inovação, Campinas, v. 2, n. 3, p. 26-27, 2006.

GRUPP, H. Foundations of the economics of innovation. Theory, measurement and practice. Massachusetts: Edward Elgar Publishing, 1998.

HINLOOPEN, J. Innovation performance across europe. Econ. Innov. New Techn, v.12, n.3, p. 145-161, 2003.

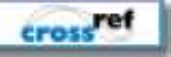

HODGSON, Geofrey M. Institutional economics: surveying the "old" and the "new". Metroeconomica. v. 44, n. 1, p. $1-28,1993$.

cross ref

HU, A. G. R\&D Organization, monitoring intensity and innovation performance in chinese industry. Econ. Innov. New Techn, v. 12, n. 2, p. 117-144, 2003.

cross ref

INÁCIO JÚNIOR, E.; QUADROS, R. Apresentando a fórmula do IBI. Revista Inovação Uniemp, São Paulo, v. 2 , n. 5 , p. 26 - 27, nov. 2006.

LAURSEN, K.; SALTER, A. Open for innovation: the role of openness in explaining innovation performance among U.K. manufacturing firms. Strategic Management Journal, v. 27, n. 2, p. 131-150, 2006.

cross ref

LEIPONEN, A. Why do firms not collaborate? The role of competencies and technological regimes. In.

KLEINKNECHT, A. MOHNEN, P. Innovation and firm performance. Econometric explorations of survey data. New York: Palgrave, 2002.

MANGEMATIN, V.; MANDRAN, N. Do non-R\&D intensive industries benefit from public research spillovers? The case of the agro food industry. In. KLEINKNECHT, A. MOHNEN, P. Innovation and firm performance. Econometric explorations of survey data. New York: Palgrave, 2002.

MANUAL DE OSLO. Proposta de Diretrizes para Coleta e Interpretação de Dados sobre Inovação Tecnológica. Rio de Janeiro: FINEP, 2004.

MARCOVITCH, J. Interação da instituição de pesquisa industrial com seu ambiente e suas implicações na eficácia organizacional. 1978. 680f. Tese (Livre Docência) - Faculdade de Economia, Administração e Contabilidade, Universidade de São Paulo, São Paulo, 1978. 
MARQUES, P. S. M. Estrutura de Capital e Estratégia de Inovação: Um Estudo no Contexto Brasileiro. 2007. $111 \mathrm{f}$. Dissertação (Mestrado em Administração de Empresas) Departamento de Administração, Universidade Presbiteriana Mackenzie. São Paulo, 2007.

MATESCO, V. R. Inovação tecnológica das empresas brasileiras: a diferenciação competitiva e a motivação para inovar. 1993. 384f. Tese (Doutorado em Economia) - Instituto de Economia Industrial, Universidade Federal do Rio de Janeiro, Rio de Janeiro, 1993.

MOHNEN, P.; MAIRESSE, J.; DAGENAIS, M. Innovativity: A Comparisson Across Seven European Countruies. Econ. Innov. New Techn., v. 15, n. 4-5, p. 391-413, junho/julho 2006.

MORGANTI, F. Inovação Tecnológica e Desempenho das Empresas do Setor Químico no Brasil. 2005.155 f. Dissertação (Mestrado em Administração de Empresas) - Programa de Pós Graduação em Administração de Empresas, Universidade Presbiteriana Mackenzie, São Paulo, 2005.

NELSON, R. R.; DOSI, G. An introduction to evolutionary theories in economics. Journal of Evolutionary Economics., v. 4, n. 3, p. 153-172, Springer/1994.

PAULINYI, E.I. Padrões de capacitação tecnológica da empresa. Revista da Administração da USP (RAUSP), v. 25, n. 4, p. 66-71, out./dez. 1990.

PINTEC. Pesquisa de Inovação Tecnológica. Rio de Janeiro: IBGE, 2002.

PINTEC. Pesquisa de Inovação Tecnológica. Rio de Janeiro: IBGE, 2005.

PINTEC. Pesquisa de Inovação Tecnológica. Rio de Janeiro: IBGE, 2007.

ROS, E. M.; LABEAGA, J. M. Modelling innovation activities using discrete choice panel data models. In. KLEINKNECHT, A. MOHNEN, P. Innovation and firm performance. Econometric explorations of survey data. New York: Palgrave, 2002.

SAMPSON, R. C. R\&D Alliances and firm performance: the impact of technological diversity and alliance organization on innovation. Academy of Management Journal., v. 50, n. 2, p. 364-386, 2007.

SANTOS, D. F. L. A influência da inovação no desempenho das firmas no Brasil. 2009. 315f. Tese (Doutorado em Administração de Empresas) - Programa de Pós-Graduação em Administração de Empresas, Universidade Presbiteriana Mackenzie, São Paulo, 2009.

SANTOS, D. F. L.; POPADIUK, S. Influência do capital humano no sistema de inovação da firma: a formação de um construto. Revista Organizações em Contexto, São Bernardo do Campo, v. 7, n. 13, p. 95-115, jan./jun. 2011.

SBRAGIA, R. (Coord.). Inovação: como vencer esse desafio empresarial. São Paulo: Clio Editora, 2006.

SCHUMPETER, J. A. A Teoria do Desenvolvimento Econômico: uma investigação sobre lucros, capital, crédito, juro e ciclo econômico. 2. ed. São Paulo: Nova Cultural, 1985.

SILVA, S. T. On evolutionary technological change an economic growth: Lakatos as a starting point for appraisal. Porto: Faculdade de Economia do Porto, 2004. (Working papers, 139).

SOETE, L.; FREEMAN, C. The economics of industrial innovation. Cambridge: MIT Press, 1999.

SUNDBO, J. The theory of innovation. Entrepreneurs, technology and strategy. Cheltenham: Edward Elgar Publishing, 1998.

TIDD, J.; BESSANT, J.; PAVITT, K. Gestão da Inovação. 3. ed. Porto Alegre: Bookman, 2008.

\section{Dados dos autores:}

Nome Completo: David Ferreira Lopes Santos 
Filiação institucional: Universidade Estadual Paulista - FCAV Jaboticabal/SP

Departamento: Economia Rural

Função ou cargo ocupado: Professor Assistente Doutor

Endereço completo para correspondência (bairro, cidade, estado, país e CEP): Via de Acesso Paulo

Donato Castelanne S/N - UNESP - Depto. Economia Rural. Jaboticabal-SP: CEP: 14.884-900

Telefones para contato: (16) 3209-2634

e-mail: david.lopes@fcav.unesp.br

Recebido em: $21 / 05 / 2012$

Aceito em: 24/09/2012 